\title{
EIGENFUNCTION EXPANSIONS AND SIMILARITY FOR CERTAIN NONSELFADJOINT OPERATORS ${ }^{1}$
}

\author{
BY CHARLES GOLDSTEIN
}

\author{
Communicated by M. H. Protter, January 7, 1969
}

1. Let $A_{0}$ denote a selfadjoint operator acting in a Hilbert space $H$ with spectral resolution $E_{0_{\lambda}}$. We assume that for a fixed open interval $S$, the operator $A_{0} E_{0 S}$ has an absolutely continuous spectrum with uniform spectral multiplicity. Let $A=A_{0}+V$ be a closed operator acting in $H$ such that $D(V) \supseteq D\left(A_{0}\right)=D(A)=D\left(A^{*}\right) \subseteq D\left(V^{*}\right)$ and $A^{*}=A_{0}+V^{*}$. Suppose that there exists an $\epsilon_{0}$ such that for $0<\epsilon<\epsilon_{0}$ and $\lambda$ in $S$, we have $\lambda \pm i \epsilon \in \rho(A), \rho(A)$ denoting the resolvent set of $A$. We wish to construct two complete, orthonormal sets of eigenfunctions for the continuous part, $A^{c}$, of the operator $A$ given a complete, orthonormal set of eigenfunctions for $A_{0}^{c}$.

We first define the notion eigenfunction expansion. Suppose that $B$ is a locally convex linear topological space, dense in $H$, such that the injection mapping from $B$ into $H$ is continuous. Let $B^{*}$ denote the dual space of $B .(,)_{H}$ and \|\|$_{H}$ will represent the inner product and norm in $H$ and $\langle u, w\rangle$ will denote the sesquilinear pairing of $B^{*}$ and $B\left(w \in B^{*}, u \in B\right)$.

Definition. Let $(\Omega, d \omega)$ denote a $\sigma$-finite measure space. Suppose that there is a mapping $w^{0}(\lambda, \omega)$ from $S \times \Omega$ into $B^{*}$ such that the transformation $T^{0}$ given by $T^{0} u=u^{0}(\lambda, \omega)=\left\langle u, w^{0}(\lambda, \omega)\right\rangle$ for each $u$ in $B$ may be extended to an isometry from $H_{S}^{0}=E_{0 S} H$ onto $\mathfrak{H C}_{S}$ $=L_{2}(S \times \Omega)$. Also, suppose that $\left(A_{0} \imath\right)^{0}(\lambda, \omega)=\lambda \hat{\imath}^{0}(\lambda, \omega)$ for each $u$ in $D\left(A_{0}\right)$ and a.a. points $(\lambda, \omega)$ in $S \times \Omega$. We shall refer to the mappings $w^{0}(\lambda, \omega)$ as eigenfunctions and to $T^{0}$ as a spectral mapping of $A_{0} E_{0 S}$. We say that the set $\left\{w^{0}(\lambda, \omega)\right\}$ gives an eigenfunction expansion for the operator $A_{0} E_{0,5}$.

In $\$ 2$ we give sufficient conditions for the operator $A E_{S}$ to possess two eigenfunction expansions, where $E_{S}$ denotes a projection operator to be constructed presently. Employing these eigenfunctions, we establish the similarity of the operators $A_{0} E_{0_{S}}$ and $A E_{S}$ as well as a time-dependent scattering theory. In $\$ 3$ we apply these results to differential operators as well as to "gentle" perturbations. In this note we confine ourselves to the statement of results. All of the results will be proven in a subsequent publication.

1 Work performed under the auspices of the U. S. Atomic Energy Commission. 
2. Suppose that for $0<\epsilon \leqq \epsilon_{0}$ and $\lambda$ in $S$, the operators $q(\lambda \pm i \epsilon)$ $=I-V R(\lambda \pm i \epsilon) \quad\left(p(\lambda \pm i \epsilon)=I-V^{*} R(\lambda \pm i \epsilon)^{*}\right)$ are continuous and invertible on $B$. We suppose that there exist continuous invertible operators $q(\lambda \pm i 0)(p(\lambda \pm i 0))$ defined on $B$ such that $q(\lambda \pm i \epsilon)(p(\lambda \pm i \epsilon))$ and $q^{-1}(\lambda \pm i \epsilon)\left(p^{-1}(\lambda \pm i \epsilon)\right)$ converge strongly in $B$ to $q(\lambda \pm i 0)(p(\lambda \pm i 0))$ and $g^{-1}(\lambda \pm i 0)\left(p^{-1}(\lambda \pm i 0)\right)$, respectively, as $\epsilon \downarrow 0$. We also assume that for each $u$ and $v$ in $B$ and $\lambda$ in $S$,

$$
\begin{aligned}
\lim _{\epsilon \downarrow 0}\left(E_{0}^{\prime}(\lambda, \epsilon) u, v\right)_{H} & =\lim _{\bullet \downarrow 0}\left(\frac{1}{2 \pi i}\left(R_{0}(\lambda+i \epsilon)-R_{0}(\lambda-i \epsilon)\right) u, v\right)_{H} \\
& =\frac{d}{d \lambda}\left(E_{O(-\infty, \lambda)} u, v\right)_{H}=\left\langle E_{0}^{\prime}(\lambda) u, v\right\rangle
\end{aligned}
$$

defines a continuous sesquilinear form on $B$.

Define

$$
w^{ \pm}(\lambda, \omega)=p(\lambda \pm i 0)^{*} w^{0}(\lambda, \omega)
$$

and

$$
w^{\prime \pm}(\lambda, \omega)=q(\lambda \mp i 0) w^{0}(\lambda, \omega) .
$$

Note that $w^{ \pm}(\lambda, \omega)$ and $w^{ \pm}(\lambda, \omega) \in B^{*}$. Set

$$
T^{ \pm} u(\lambda, \omega)=\mathfrak{u}^{ \pm}(\lambda, \omega)=\left\langle u, w^{ \pm}(\lambda, \omega)\right\rangle
$$

and

$$
T^{\prime \pm} u(\lambda, \omega)=a^{\prime \pm}(\lambda, \omega)=\left\langle u, w^{\prime \pm}(\lambda, \omega)\right\rangle,
$$

$u, v$ in $B$. We assume that $T^{ \pm}$and $T^{\prime \pm}$ may be extended to continuous mappings from $H$ into $\mathcal{H}_{s}$. We now have

THEOREM 1. For each bounded open subset $S_{1}$ of $S$, there exists a continuous linear operator $E_{S_{1}}$ acting in $H$ such that for $u, v$ in $H$ we have

(a) $\left(E_{S_{1}} u, v\right)_{H}=\lim _{\epsilon \downarrow \downarrow} \int_{S_{1}}\left(E^{\prime}(\lambda, \epsilon) u, v\right) d \lambda=\int_{S_{1}} a^{\prime+}(\lambda, \omega) \overline{\hat{v} \pm(\lambda, \omega)} d \omega d \lambda$.

(b) $E_{S_{1}} E_{S_{2}}=E_{S_{1} \cap S_{2}}$, where $S_{2}$ is an arbitrary bounded, open subset of $S$. If $S_{1} \cap S_{2}=\phi$, then $E_{S_{1}}+E_{S_{2}}=E_{S_{1} \cup S_{2}}$.

(c) $E_{S_{1}} A \subseteq A E_{S_{1}}$.

(d) $T^{+*} T^{++}=E_{S}$ and $T^{\prime+*} T^{+}=E_{S}^{*}$.

Suppose that $u, v \in H$. We define continuous operators $W^{ \pm}\left(W^{\prime \pm}\right)$ from $H$ into $H$ by $W^{ \pm}=T^{ \pm *} T^{0}\left(W^{ \pm}=T^{ \pm *} T^{0}\right)$.

TheOREM 2. Suppose that $T^{ \pm}$and $T^{\prime \pm}$ map onto all of $\mathfrak{H C}_{\mathrm{S}}$. Then

(a) the mappings $T^{ \pm}\left(T^{\prime}\right)$ have continuous inverses $T^{ \pm^{-1}}\left(T^{ \pm^{-1}}\right)$ 
defined on $\mathfrak{H C}_{S}$. We have $T^{ \pm^{-1}}\left(T^{ \pm^{-1}}\right)=T^{\prime \pm^{*}}\left(T^{ \pm^{*}}\right)$.

(b) $\left(E_{S_{1}} u\right)^{\prime \hat{x}}(\lambda, \omega)=\chi_{s_{1}}(\lambda) \mathfrak{u}^{\prime \pm}(\lambda, \omega)$ and

$$
\left(E_{S_{1}}^{*} u\right)^{\hat{ \pm}}(\lambda, \omega)=\chi_{s_{1}}(\lambda) \hat{u}^{ \pm}(\lambda, \omega),
$$

where $u \in H$ and $\chi_{s_{1}}(\lambda)$ denotes the characteristic function of the set $S_{1}$.

(c) $W^{ \pm}\left(W^{ \pm}\right)$maps $E_{0 S} H$ onto $E_{S} H$. Also $W^{ \pm-1} A E_{S} W^{ \pm}=A_{0} E_{0 S}$ and $W^{ \pm^{-1}} A^{*} E_{S}{ }^{*} W^{\prime \pm}=A_{0} E_{0, S}$. Thus $A E_{S}$ and $A^{*} E_{S}^{*}$ are each similar to $A_{0} E_{0 s}$ (and hence to each other).

Our assumptions may be weakened somewhat if $A$ is selfadjoint. In this case our results are very similar to those obtained by Howland in [3], where $B$ was assumed to be a Banach space. Under additional assumptions on $E_{0}^{\prime}(\lambda)$ we may establish an analogue of Kato's theorem on the invariance of the wave operator [4]. We may also obtain explicit formulas for the scattering operator and the scattering matrix in terms of transmission and reflection coefficients. The details will appear elsewhere.

3. Denote by $A_{0}\left(A_{\epsilon}=A_{0}+\epsilon V\right)$ the closure of the elliptic partial differential operator $-\Delta\left(-\Delta+\epsilon \sum_{|\alpha|_{\leq 2}} a_{\alpha}(x) D^{\alpha}\right)$ acting on $C_{0}^{\infty}\left(R^{N}\right)$, where $R^{N}$ denotes $N$-dimensional Euclidean space $(N \geqq 2), x$ $=\left(x_{1}, x_{2}, \cdots, x_{N}\right)$ represents an arbitrary point in $R^{N}, \epsilon$ denotes a complex number, and the coefficients $a_{\alpha}(x)$ are sufficiently smooth and have bounded support. Employing the results of $\$ 2$ as well as elliptic estimates, we obtain

THEOREM 3. The results of $\$ 2$ are applicable to $A_{0}$ and $A_{6}$ with respect to a bounded interval $S$ provided any of the following conditions hold.

(a) $\epsilon=1, V$ is selfadjoint, and $S$ contains no eigenvalues of $A_{\epsilon}$.

(b) No $\lambda$ in $S$ is an accumulation point of nonreal points in the spectrum of $A_{\epsilon}$, and for no $\lambda$ in $S$ is there a nontrivial solution $u(x)$ of the equation $\left(-\Delta+\epsilon \sum_{|\alpha| \leq 2} a_{\alpha}(x) D^{\alpha}-\lambda\right) u(x)=0$ such that $u(x)$ satisfies certain outgoing or incoming radiation conditions at infinity. The same conditions hold for $A_{:}^{*}$.

(c) $|\epsilon|$ is sufficiently small.

The operators $-\Delta+q\left(-d^{2} / d x^{2}+q\right)$, may also be treated using these methods, where $q$ denotes multiplication by a complex valued potential $q(x)$ (not necessarily with bounded support) defined in $R^{N}, N \geqq 2(N=1)$. For $N=3$ this problem was considered by Mochizuki [5]. Results similar to Theorem 3 also hold for differential operators acting in various unbounded domains contained in $R^{N}, N \geqq 1$. See [1] and [2] for the selfadjoint case. 
Finally we remark that our methods are applicable to gentle perturbations. We obtain extensions of certain results due to Mochizuki [6], concerning nonselfadjoint gentle perturbations of a selfadjoint operator. A complete treatment of these and related problems will appear elsewhere.

\section{REFERENCES}

1. C. Goldstein, Eigenfunction expansions associated with the Laplacian for certain domains with infinite boundaries. I, Trans. Amer. Math. Soc. 135 (1969), 1-31.

2. - Eigenfunction expansions associated with the Laplacian for certain domains with infinite boundaries. II. Trans. Amer. Math. Soc. 135 (1969), 33-50.

3. J. S. Howland, $A$ perturbation theoretic approach to eigenfunction expansions, J. Functional Analysis 2 (1968), 1-23. 1966.

4. T. Kato, Perturbation theory for linear operators, Springer-Verlag, New York,

5. K. Mochizuki, Eigenfunction expansions associated with the Schrödinger operator with a complex potential and the scattering inverse problem, Proc. Japan Acad. 43 (1967), 638-643.

6. - On the large perturbation by a class of non-selfadjoint operators, J. Math. Soc. Japan 19 (1967), 123-158.

BrookhaVen National Laboratory, Upton, New York 11973 